\title{
MECHANICAL ASPECTS OF ANTI-CORROSIVE COATINGS PERFORMANCE TESTS
}

\author{
Alex A. Volinsky \\ Email: volinsky@eng.usf.edu \\ University of South Florida, \\ Department Mechanical Engineering \\ 4202 E Fowler Ave ENB118, \\ Tampa FL 33620, \\ USA
}

\begin{abstract}
Protective coating corrosion failure is a complicated process that involves various phenomena which are hard to test for separately. An adequately protected pipe can be in service for a relatively long time without exhibiting any signs of corrosion failure, and then all of a sudden it corrodes over a short period of time. Failure will definitely happen if the interface between the pipe surface and its protective coating system is somehow compromised or exposed to the environment. While corrosion reactions have quite a bit of variability in terms of time, the situation is further complicated when corrosion is coupled with the protective coating mechanical failure. Similar to materials fracture, corrosion failures are governed by the laws of probability, where multiple variables control the ultimate outcome.

The coating system needs to be adequately tested before placing it in service, so there is a strong need for a meaningful corrosion test. A typical test would incorporate exposing scratched coating surface to certain corrosive environments for a fixed amount of time, trying to simulate the real conditions as close as possible. Sometimes, a so-called "freeze" step is introduced to "stress" the coating prior to exposing it to the test environment, ultimately causing its failure. Due to the differences in thickness, elastic, thermal and adhesion properties, various coatings would exhibit different results when exposed to a fixed freeze temperature. One needs to understand the mechanics of the freeze in order to properly utilize it. This paper considers the coating delamination and fracture mechanics, including the freeze step mechanics in terms of the strain energy release rate, and coating sub-critical debonding. This research is sponsored by the NACE University Research Seed Grant.
\end{abstract}

Key Words: Anti-Corrosive Performance Tests, Thermal Cycling, Freeze Step, Fracture Mechanics. 


\section{INTRODUCTION}

There is a large variety of corrosion protective coating systems. They all serve the main ultimate goal of long-term corrosion protection. One has to realize though that due to the nature of the corrosion process, which brings the material to a lower energy, thermodynamically favorable state, most coatings will eventually fail, and the material they are protecting will corrode. Figure 1 shows examples of vintage mirror silver backing failures. Although the coatings do not serve the purpose of corrosion protection in this case, they probably set some of the oldest examples of mass-produced coatings failures. Even nowadays mirror coatings are prone to failures, presenting a technological challenge [1].
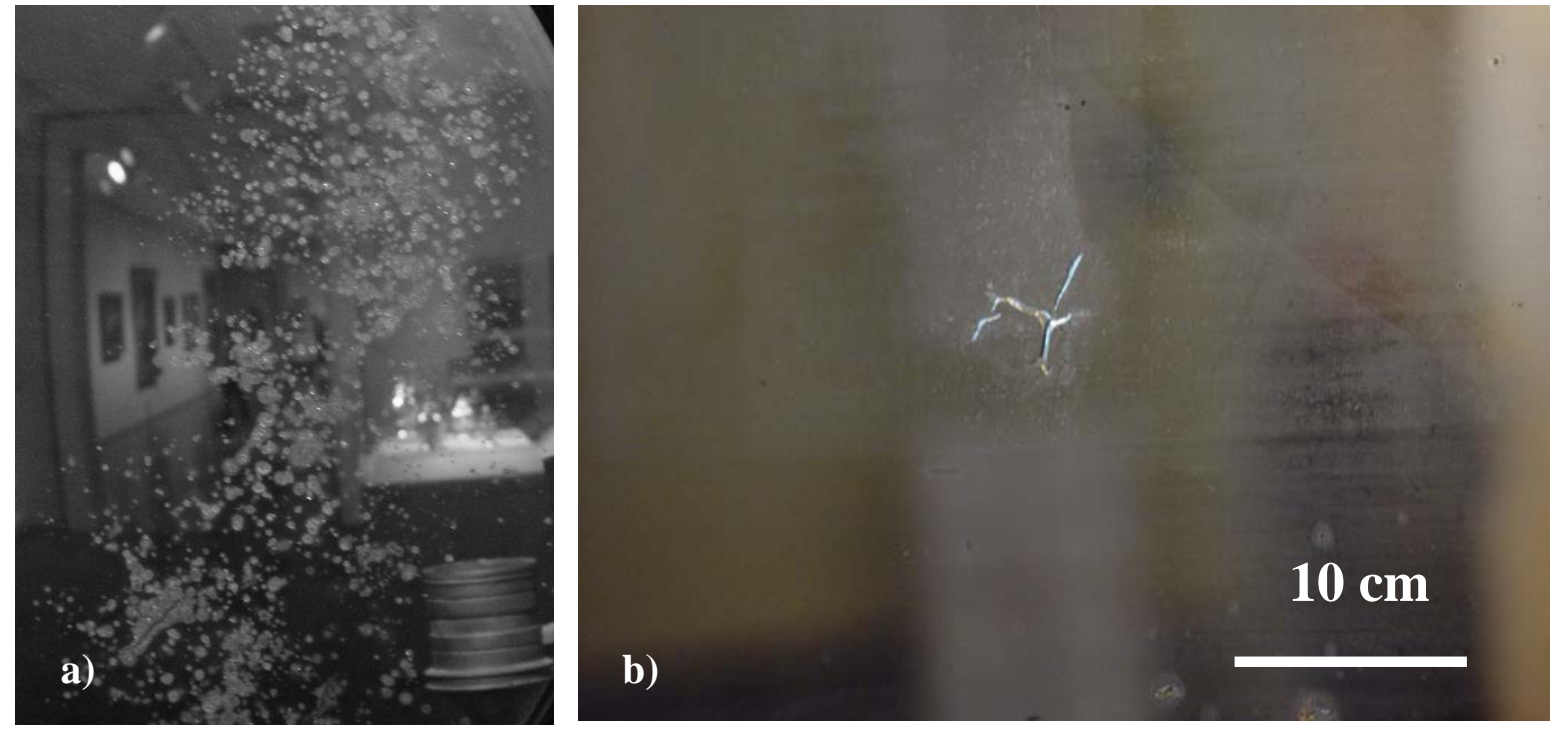

FIGURE 1. a) Mirror backing corrosion failure. The Art Institute of Chicago, circa 1820; b) Buckling mirror silver backing delamination failure, Correr Museum, Venice, Italy, $18^{\text {th }}$ century.

Unfortunately, premature failures can be costly, especially when the offshore applications are considered. It is extremely important to test corrosion protective coatings before they are deployed. Various tests have been designed for evaluating coatings performance. See reference [2] for a comprehensive review, which also inspired this publication.

Anti-corrosive performance testing serves the purpose of comparison and ranking the coatings, as well as eliminating the ones likely to exhibit premature failure. Unfortunately there is no single test available, capable of fully predicting protective coatings performance and their lifespan. Although, based on a combination of tests, one can make an assessment and compare various coatings' performance. It is important to understand how the coating properties influence test outcomes, so that they will be properly interpreted. Here, we address the mechanical aspects of anti-corrosive coatings performance tests. 


\section{MECHANICAL COATING FAILURE}

A coating will fail to provide anti-corrosion protection when it fractures or de-adheres (delaminates). By fracture we mean the formation of through-thickness cracks in the coating, which may or may not extend all the way to the substrate (Figure 2a). Cohesive or interfacial delamination exposes protected material to the environment (Figure 1b), while adhesive fracture leaves a weak spot, leading to stress concentrations and long-term corrosion problems, shortening the protected part lifetime. Both cohesive and adhesive failures can be treated similarly from the mechanics perspective. Thermal cycling resistance, dimensional stability, and flexibility tests are performed to address the issues of coating fracture and adhesion loss. Thermal cycling resistance test is qualitative, i.e. the sample is subjected to thermal cycling, after which the coating is inspected for cracks. Flexibility and dimensional stability tests are semi-quantitative.
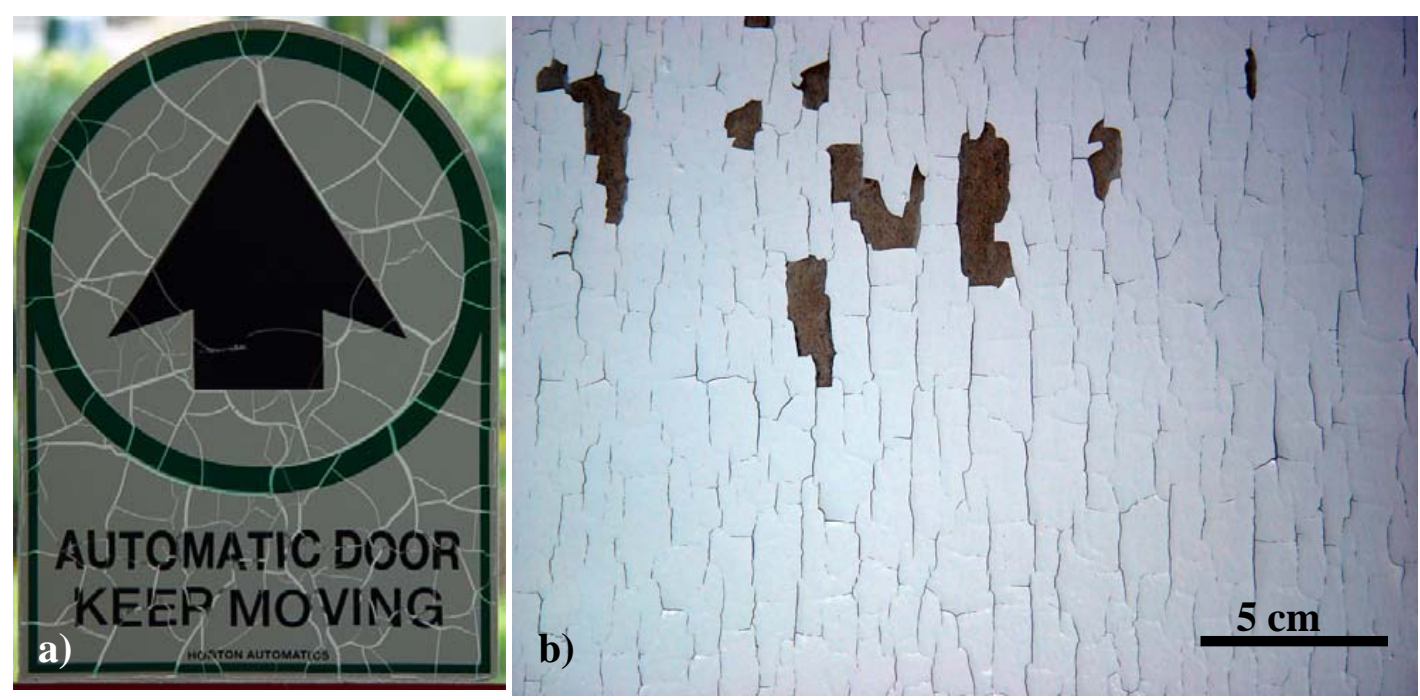

FIGURE 2. a) Through-thickness cracks in a polymer film adhered to glass; b) Cracking and delamination of organic paint.

Let's consider mechanical aspects of a coating delamination and fracture processes. The strain energy release rate, $G$, of a stressed coating is [3]:

$$
G=Z \frac{\left(1-v_{c}^{2}\right) \sigma^{2} t}{E_{c}}
$$

where $\sigma$ is the stress in the coating, $t$ is its thickness, or the thickness of the delaminated film portion in the case of adhesive failure, $v_{c}$ and $E_{c}$ are its Poisson's ratio and elastic modulus, respectively, and $\mathrm{Z}$ is a dimensionless cracking parameter. Coating will delaminate when the strain energy release rate, $G$, exceeds the interfacial toughness, $\Gamma_{i}(\Psi)$ :

$$
G \geq \Gamma_{i}(\Psi)
$$


Here, $\Psi$ is the crack phase angle, which is $0^{\circ}$ for the mode $\mathrm{I}$, and $90^{\circ}$ for the mode II loading. The coating will crack when the strain energy release rate exceeds the coating toughness, $\Gamma_{\text {coating: }}$ :

$$
G \geq \Gamma_{\text {coating }}(\Psi)
$$

This case applies to both through-thickness (Figure 2a) and adhesive coating failures.

In a quantitative adhesion or fracture test, the amount of energy necessary to delaminate or fracture a square area of a coating is measured. Energy-based tests have advantages over the stress or force-based tests, as they account for the stress concentration effects. While there are several quantitative adhesion and coating toughness tests available, they are not widely applied for the anticorrosive coatings, possibly due to complications with tests administration and data interpretation. There are many parameters, affecting the measurements. For example, according to equation 1, a thicker coating would more likely delaminate or fracture compared to a thinner one. A quantitative adhesion test would give different results for different thickness coatings, so correct interpretation is always necessary.

While thin film stress measurements based on the substrate curvature methods are performed routinely in the microelectronics industry, stress characterization is less common in the anti-corrosion coating industry, and the measurement methodology has been implemented only recently [3, 5]. Although stress levels in anti-corrosive coatings are orders of magnitude lower [3], compared to thin films in microelectronics (MPa vs. GPa), this is offset by the lower coating modulus and larger thickness in terms of the strain energy release rate (equation 1). A $1 \mu \mathrm{m}$ thick tungsten film on a ceramic substrate with $1 \mathrm{GPa}$ compressive residual stress would have the same amount of stored elastic energy per unit area, as a $200 \mu \mathrm{m}$ thick polyamide coating with $5 \mathrm{MPa}$ residual tensile stress. Let's consider the mechanics of a freeze step in the anti-corrosive coating test, when the coating is scratched prior to low temperature exposure.

\section{FREEZE STEP MECHANICS}

The freeze step causes extra tensile stress in the coating at lower temperatures, since its thermal expansion coefficient is typically larger than that of a protected substrate (Ex. polymer coating on a metal substrate). The biaxial coating stress caused by lowering the temperature by $\Delta \mathrm{T}$ can be estimated as:

$$
\sigma_{\text {thermal }}=\frac{E_{c}}{1-v_{c}}\left(\alpha_{c}-\alpha_{s}\right) \cdot \Delta T
$$

where $\alpha_{c}$ and $\alpha_{s}$ are thermal expansion coefficients of the coating and the substrate, respectively.

Since the coating is scratched prior to a freeze step, the stress at the crack tip due to the scratch will be much larger than the thermal stress in equation 4 (stress concentration):

$$
\frac{\sigma_{\text {cracktip }}}{\sigma_{\text {thermal }}}=C \sqrt{\frac{a}{\rho}}
$$


Here, $C$ is a non-dimensional geometrical loading parameter, $a$ is the crack length, and $\rho$ is the crack tip radius. Since the crack length is much larger than its tip radius, orders of magnitude stress magnification is realistic. If one were to consider a $100 \mu \mathrm{m}$ interfacial pre-crack, introduced by the scratching process, with a large tip radius of $1 \mu \mathrm{m}$, then the stress at the crack tip will be magnified by 10 times, resulting in a 100 -fold increase of the strain energy release rate in equation 1 . Without considering the fact that materials and interfaces are more brittle at lower temperatures (lower $\Gamma_{i}(\Psi)$ and $\Gamma_{\text {coating }}(\Psi)$ ), the conditions of equations 2 and 3 are more likely to be met in this case, and the coating will delaminate around the scratch, causing consequent corrosion failures described in [2]. In addition to a scratch, any other defect, even of atomic dimensions, will act as a stress concentrator.

When ranking the coatings based on the test employing a freeze step one has to take into account the above mechanics, as various coatings would have different thermal expansion coefficients, elastic moduli and thickness. For the same temperature decrease and time exposure of the freeze step, the stress developed in the castings will be different, according to equation 4, thus leading to a different strain energy release rate (combining equations 1 and 4):

$$
G=Z \frac{\left(1+v_{c}\right) E_{c}\left(\alpha_{c}-\alpha_{s}\right)^{2} \Delta T^{2} t}{\left(1-v_{c}\right)}
$$

Even if a coating is partially delaminated, it will fail to provide corrosion protection, and if there is no crack growth during the freeze step, higher stress concentration at the scratch or defect site will eventually lead to corrosion. Consequent temperature cycling is detrimental to corrosion protection. Figure 3 shows polymer film buckling delamination and through-thickness cracking on metal substrates of street signs. This happens due to daily and seasonal temperature fluctuations, leading to corrosion, presented in Figure 3b. While the stress is uniformly distributed in a freshly-applied polymer coating, it redistributes due to thermal cycling, forming local regions of tensile and compressive local stresses (Figure 3c), which ultimately causes coating failure.

One of the quantitative adhesion tests, modified edge lift-off test (MELT) is based on the fact that a polymer coating shrinks more, peeling the film of interest underneath it upon cooling [6]. One needs to keep in mind that materials and interfaces are more brittle at lower temperatures, so the adhesion values obtained by this test should be considered as a lower bound. Other popular quantitative adhesion tests are the four-point bend [7], and the superlayer indentation tests [8]. In addition to measuring interfacial fracture toughness, indentation methods are also capable of assessing thin film and coating toughness $[9,10]$.

Recently, we have modified the superlayer indentation test for measuring thin film adhesion in moist environments [11]. Up to a 100-fold decrease in adhesion due to water presence was measured for $\mathrm{Cu}$ and diamond-like carbon (DLC) thin films [12]. Even without considering corrosion effects, we believe that water reduces the surface energy of the newly-formed surfaces during the fracture process, effectively reducing the interfacial and coating fracture toughness, making it easier to satisfy the fracture condition of equations 2 and 3. Corrosion environments would have even more pronounced effects. 

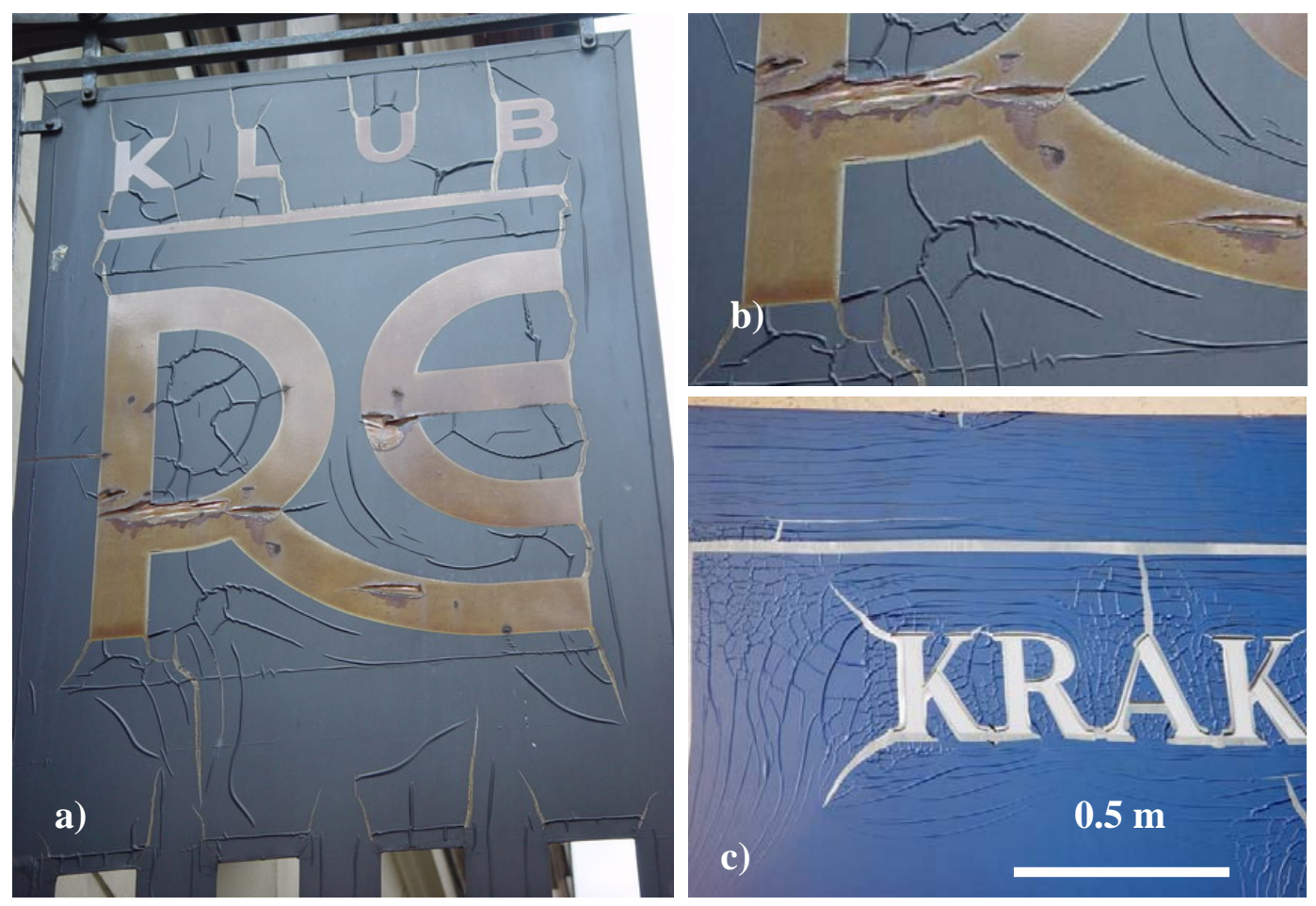

FIGURE 3. a) and b) Polymer film delamination and fracture on a street sign, with b) the brass substrate corrosion; c) Buckling and cracking of a polymer film on Al substrate.

\section{CONCLUSIONS}

Mechanical aspects of anti-corrosion protective coating tests have been considered with an emphasis on a freeze step. One has to be careful in comparing the test results, as the coating thickness, elastic modulus, and thermal expansion coefficient need to be taken into account. Reducing the number and the amplitude of the temperature cycles, keeping the interface free of moisture, reducing the film thickness and stress levels would be good measures to reduce and prevent failures in the field. While progress is anti-corrosive coating testing methodologies is noticeable, an ultimate corrosion/adhesion test is yet to be developed.

\section{ACKNOWLEDGEMENTS}

The author would like to acknowledge the financial support for this research from NACE International under contract N000140210024. P.J. Waters work on thin film adhesion measurements in wet environments is also greatly appreciated. 


\section{REFERENCES}

1. V.P. Burolla, "Deterioration of the Silver/glass Interface in Second Surface Solar Mirrors”, Solar Energy Materials, Vol. 3/1-2 (1980), pp. 117-126.

2. M.J. Mitchell, D. Claydon, D. Ward, "A Critical Review of Current Performance Tests for Offshore Anti-corrosive Coatings”, Corrosion '05, NACE Paper No. 05021.

3. J.W. Hutchinson; Z. Suo, "Mixed-mode Cracking in Layered Materials", Advances in Applied Mechanics 29 (1992), pp. 63-191.

4. Y. Korobov, D.P. Moore, “Performance Testing Methods for Offshore Coatings: Cyclic, EIS and Stress”, Corrosion ’04, NACE Paper No. 04005.

5. ASTM D6991-05, “Measurements of Internal Stresses in Organic Coatings by Cantilever (Beam) Method”, ASTM International

6. G. Charalambides, J. Lund, A.G. Evans, R.M. McMeeking, “A Test Specimen for Determining the Fracture Resistance of Bimaterial Interfaces”, J. Appl. Mech. 111 (1989), pp. 77-82.

7. T.W. MacElwee, I.D. Calder in: C.M. Osburn and J. Andrews (Eds.), J. Electrochem. Soc. Proc. Second International Symposium on Ultra Large Scale Integration Science and Technology 89-9 (1989), p. 693.

8. A.A. Volinsky, N.R. Moody, W.W. Gerberich, "Interfacial Toughness Measurements for Thin Films on Substrates”, Acta Mater. Vol. 50/3 (2002), pp. 441-466.

9. A.A. Volinsky, J.B. Vella, W.W. Gerberich, "Fracture Toughness, Adhesion and Mechanical Properties of Low-K Dielectric Thin Films Measured by Nanoindentation”, Thin Solid Films 429/1-2, (2002), pp. 201-210.

10. J.B. Vella, I.S. Adhihetty, K. Junker, and A.A. Volinsky, "Mechanical Properties and Fracture Toughness of Organo-silicate Glass (OSG) Low-k Dielectric Thin Films for Microelectronic Application”, International Journal of Fracture, Vol. 119, No. 4, (2003), pp. 487-499.

11. A.A. Volinsky, P.J. Waters, "Novel Adhesion Test for Environmentally Assisted Fracture in Thin Films”, (2005), Tri-Service Conference on Corrosion, November 14-18, 2005, Orlando, FL Proceedings Paper 104.

12. P.J. Waters, A.A. Volinsky, "Moisture Effects on Copper Thin Film Adhesion", Proceedings of IMECE: ASME 2005 (Orlando, FL, Nov. 5-11, 2005), paper 81532. 